\title{
Febrile Morbilliform Rash as a Clinical Presentation of COVID-19 in a Pediatric Patient
}

\author{
Muneer Almutairia, c, Amani Azizalrahman ${ }^{\mathrm{b}}$
}

\begin{abstract}
The emergence of severe acute respiratory syndrome coronavirus 2 (SARS-CoV-2) in January 2020 resulted in a worldwide public health emergency. While the pediatric age group currently includes no more than $2 \%$ of the population diagnosed with coronavirus disease 2019 (COVID-19), the clinical manifestations in this cohort can range from upper respiratory tract symptoms to respiratory system and multiorgan failure. Interestingly, most of the pediatric COVID-19 patients are asymptomatic at the time of presentation. As such, the full clinical spectrum of this disease remains unknown. We describe here the case of a previously healthy 16-month-old boy who presented with a threeday history of febrile morbilliform exanthematous rash associated with confirmed COVID-19 infection. This case presentation serves to expand our knowledge base with respect to the diverse presentations of this highly contagious illness, particularly among pediatric patients. We recommend maintaining a high index of suspicion when evaluating children who present with rash and fever during the current pandemic of COVID-19.
\end{abstract}

Keywords: COVID-19; Morbilliform rash; Fever; Pediatric; Febrile; SARS-COV-2

\section{Introduction}

The emergence of severe acute respiratory syndrome coronavirus 2 (SARS-CoV-2) resulted in a worldwide pandemic and public health emergency [1]. While pediatric patients currently represent no more than $2 \%$ of those diagnosed with coronavirus disease 2019 (COVID-19), clinical manifestations in this cohort can range from upper respiratory tract symptoms to

Manuscript submitted September 9, 2020, accepted September 18, 2020

Published online November 3, 2020

aPediatric Neurology Department, National Neurosciences Institute, King Fahad Medical City, Riyadh, Saudi Arabia

bPediatric Emergency Medicine Department, King Fahad Medical City, Riyadh, Saudi Arabia

${ }^{\mathrm{c} C}$ Corresponding Author: Muneer Almutairi, Pediatric Neurology Department, National Neurosciences Institute, King Fahad Medical City, Riyadh, Saudi Arabia. Email: mmalmutairi@kfmc.med.sa

doi: https://doi.org/10.14740/ijcp408 respiratory system and multiorgan failure $[1,2]$. Most pediatric patients are asymptomatic at presentation. Although the population of pediatric COVID-19 patients has expanded, the full clinical spectrum of this condition remains unknown [2].

We describe here a case of COVID-19 in a pediatric patient who presented for evaluation and treatment of fever and an associated exanthematous rash.

\section{Case Report}

A 16-month-old male presented to the Pediatric Emergency Department at King Fahad Medical City, Riyadh, Saudi Arabia with a three-day history of high fever and rash with irritability.

He was born at full term to healthy non-consanguineous Saudi parents; he was fully immunized and was previously healthy. The parents reported sudden and simultaneous onset of both fever and rash. His fever was high, reaching a maximum of $39{ }^{\circ} \mathrm{C}$, recurred intermittently over the course of 3 days, and was responsive to antipyretics. The rash was diffusely pinkish to red and pruritic and progressed in a cephalocaudal direction; it involved the face only on the first day, followed by the neck, trunk and extremities over the course of the 2 days to follow (Fig. 1). Alongside with it, a honeycolored and crusty lesion on an erythematous base measuring $1 \times 1 \mathrm{~cm}$, in consistence with impetigo, was evidently present on the lower back. No rash was detected on the palms or soles of feet, and there was no evidence of desquamation; there was no associated eye redness, or mouth or genital lesions. Apart from the fever and rash, he was mildly dehydrated otherwise active and able to take food and water orally.

The child lives with his family which consists of six members, of whom none developed any symptoms. The parents denied any contact with any individuals who were ill or had similar symptoms during the 2 months prior to presentation; they were self-isolating during the COVID-19 pandemic, and noted that no one was leaving the home for school or work. There was also no history of recent travel; no one in the family was employed in the health care sector.

His diet consists of milk supplemented by soft foods, and no new food was introduced recently to his diet.

Based on the COVID-19 screening scoring system provided by the Ministry of Health, nasopharyngeal swab for SARS-CoV-2 reverse transcriptase polymerase chain reaction (RT-PCR) test was performed. No other tests were done including complete blood count, liver and renal function tests as 

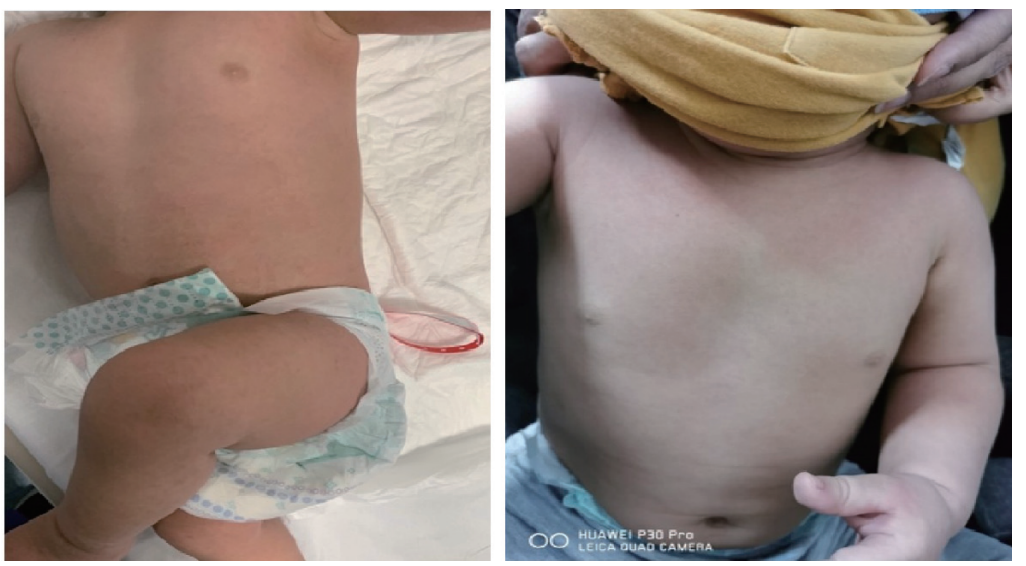

Figure 1. The exanthem consists of confluent erythematous and blanching macules and papules involving the trunk and extremities.

well as cultures, as the clinical picture was not indicative. No chest radiograph was performed as well, as he was not having any respiratory signs or symptoms.

Intravenous fluids were administered to treat acute dehydration and he was discharged to home with oral antipyretics, oral antihistamines and local antibiotics to be used to treat impetigo that had developed on the skin of his back. The family was instructed to return to the hospital after $48 \mathrm{~h}$ for a followup assessment and to review the results of the nasopharyngeal swab test.

The child and his family returned 2 days later. The exanthematous rash remained, although the lesions on his back had healed. The result of the nasopharyngeal swab test was positive for SARS-CoV-2; the child was transferred with his mother to quarantine.

Upon following this patient up, the parents reported that his fever and irritability have improved within $72 \mathrm{~h}$ of their onset, followed by a gradual improvement of the rash over 5 days after the resolution of fever.

No other family members became subsequently ill, came to be positive for SARS-CoV-2, or developed any similar presentations.

\section{Discussion}

SARS-CoV-2 is an enveloped single-stranded RNA virus that is mainly transmitted via droplets, air-borne routes and direct contact. Patients diagnosed with COVID-19 typically present with one or more of several heterogeneous manifestations that include fever, dry cough, headache and/or myalgias, among many others $[3,4]$.

Similar to what has been observed for other RNA viruses, SARS-CoV-2 has been associated with cutaneous manifestations [5]. While these findings remain uncommon, they include morbilliform, vesicular, urticarial, petechial or ischemic rashes, among others. Erythematous rashes are among those reported most commonly, followed by urticarial and vesicular rashes. Despite recent reports documenting the numerous dermatological manifestations associated with COVID-19, the mechanisms underlying these responses remain poorly understood [6].

There are comparatively few reports of skin rashes in pediatric COVID-19 patients [6]. Two recent reports, including one from Thailand and one from Pakistan, featured adult cases presenting with petechial rashes that were mistakenly diagnosed as dengue $[7,8]$. In another series, 22 adult patients diagnosed with COVID-19 presented with varicella-like rashes with predominant involvement of the trunk [9]. Interestingly, a recent report by Genovese et al [10] described a pediatric patient with varicella-like rash associated with the diagnosis of COVID-19. There is growing recognition of rashes as presenting symptoms among pediatric COVID-19 patients, including those with a Kawasaki-like picture or chilblain-like findings [11, 12].

Our pediatric patient had no history of drug reactions, travel history, or contact with confirmed COVID-19 patients and was up-to-date on all vaccinations; he presented with a febrile morbilliform rash and was diagnosed with COVID-19 via nasopharyngeal swab testing. The rash was intensely pruritic, and presented together with fever. The absence of mouth, palm or sole lesions, conjunctival injection or cervical lymphadenopathy made the diagnosis of measles or Kawasaki disease somewhat less likely; there remained the weak possibility of roseola infantum. While his symptoms improved in response to oral antihistamines and an oral antipyretic, the patient and his contacts were quarantined once the nasopharyngeal swab result was revealed.

The findings presented here do not explain the clinical significance of this rash in the context of COVID-19; however, they provide additional clues for the diagnosis of COIVD-19 among children. It is important to remember that pediatric SARS-CoV-2-positive patients may be mildly symptomatic or fully asymptomatic.

\section{Conclusions}

COVID-19 can be difficult to diagnose in the pediatric age group. This case expands our current knowledge regarding the diverse presentations of this highly contagious illness. We recommend maintaining a high index of suspicion when assessing a child presenting with rash and fever at this time. 


\section{Acknowledgments}

None to declare.

\section{Financial Disclosure}

The authors have no affiliations with or involvement in any organization or entity with any financial interest or non-financial interest in the subject matter or materials discussed in this manuscript.

\section{Conflict of Interest}

None to declare.

\section{Informed Consent}

Informed consent was obtained from the patient's legal guardian (his father) in order to publish this study.

\section{Author Contributions}

Conceptualization: MA and AA. Writing of original draft preparation: MA. Review and editing: MA and AA. All authors have read and agreed to the published version of the manuscript.

\section{Data Availability}

The authors declare that data supporting the findings of this study are available within the article.

\section{References}

1. Wu Z, McGoogan JM. Characteristics of and important lessons from the coronavirus disease 2019 (COVID-19) outbreak in China: summary of a report of 72314 cases from the Chinese Center for Disease Control and Prevention. JAMA. 2020;323(13):1239-1242.

2. Dong Y, Mo X, Hu Y, Qi X, Jiang F, Jiang Z, Tong S. Epidemiology of COVID-19 among children in China. Pediatrics. 2020;145(6).

3. Lu Q, Shi Y. Coronavirus disease (COVID-19) and neonate: What neonatologist need to know. J Med Virol. 2020;92(6):564-567.

4. Zhu N, Zhang D, Wang W, Li X, Yang B, Song J, Zhao X, et al. A novel coronavirus from patients with pneumonia in China, 2019. N Engl J Med. 2020;382(8):727-733.

5. Quintana-Castanedo L, Feito-Rodriguez M, ValeroLopez I, Chiloeches-Fernandez C, Sendagorta-Cudos E, Herranz-Pinto P. Urticarial exanthem as early diagnostic clue for COVID-19 infection. JAAD Case Rep. 2020;6(6):498-499.

6. Sachdeva M, Gianotti R, Shah M, Bradanini L, Tosi D, Veraldi S, Ziv M, et al. Cutaneous manifestations of COVID-19: Report of three cases and a review of literature. J Dermatol Sci. 2020;98(2):75-81.

7. Joob B, Wiwanitkit V. COVID-19 can present with a rash and be mistaken for dengue. J Am Acad Dermatol. 2020;82(5):e177.

8. Bokhari S, Mahmood F, Bokhari S. Case report: diagnosis of COVID-19 versus tropical diseases in Pakistan. Am J Trop Med Hyg. 2020;103(1):77-78.

9. Marzano AV, Genovese G, Fabbrocini G, Pigatto P, Monfrecola G, Piraccini BM, Veraldi S, et al. Varicella-like exanthem as a specific COVID-19-associated skin manifestation: Multicenter case series of 22 patients. J Am Acad Dermatol. 2020;83(1):280-285.

10. Genovese G, Colonna C, Marzano AV. Varicella-like exanthem associated with COVID-19 in an 8-year-old girl: A diagnostic clue? Pediatr Dermatol. 2020;37(3):435436.

11. Jones VG, Mills M, Suarez D, Hogan CA, Yeh D, Segal JB, Nguyen EL, et al. COVID-19 and Kawasaki disease: novel virus and novel case. Hosp Pediatr. 2020;10(6):537540.

12. Andina D, Noguera-Morel L, Bascuas-Arribas M, Gaitero-Tristan J, Alonso-Cadenas JA, Escalada-Pellitero $\mathrm{S}$, Hernandez-Martin A, et al. Chilblains in children in the setting of COVID-19 pandemic. Pediatr Dermatol. 2020;37(3):406-411. 